\title{
LUISA: Uma proposta de ferramenta para auxílio ao diagnóstico do câncer de mama a partir de imagens de mamografias digitalizadas.
}

\author{
LUISA: A breast cancer Computer-aided diagnosis proposal on scanned mammography \\ images.
}

\author{
Felipe Victor de Sá Oliveira ${ }^{1}$ (B) orcid.org/0000-0002-5913-5407 \\ Anthony Lins ${ }^{1,2}$ (D) orcid.org/0000-0002-7153-841X \\ ${ }^{1}$ Residência Tecnológica em Inteligência Artificial, Universidade de Pernambuco e FITec, Recife, Brasil,
${ }^{2}$ Universidade Católica de Pernambuco, Pernambuco, Brasil.
E-mail do autor principal: Felipe Oliveira felipe.sa.oliveirati@gmail.com
}

\section{Resumo}

Problema crescente no mundo, o câncer de mama é considerado um dos principais causadores de mortes em mulheres. A mamografia digital é o principal método de detecção precoce deste tipo de câncer, porém sua interpretação é difícil até mesmo para um profissional. Técnicas de aprendizado de máquina são utilizadas para facilitar esta interpretação. Assim, o presente trabalho tem como objetivo propor um sistema de deteç̧ão auxiliado por computador para colaborar com profissionais no diagnóstico do câncer de mama, a partir da análise de imagens de mamografias digitalizadas. Candidatos à lesão foram gerados a partir da utilização do algoritmo de detecção de ponto de interesse SURF e passaram por um processo de classificação utilizando Redes Neurais Convolucionais (CNN) e Random Forests (RF). Foram utilizadas 1210 imagens da base CBIS-DDSM. A CNN apresentou melhores resultados alcançando no treinamento 0,06\% de perda e $97 \%$ acurácia. No cenário de validação obteve $32 \%$ de perda e $93 \%$ de acurácia. Para modelo RF as características das imagens foram extraídas a partir do descritor Hu-Moments obtendo uma acurácia de $43 \% \pm 0.005$.

Palavras-Chave: câncer de mama; visão computacional; redes neurais convolucionais;

\begin{abstract}
A growing problem worldwide, breast cancer is considered a major cause of death in women. Digital mammography is the main method of early detection of this cancer, but its interpretation is difficult even for a professional. Machine learning techniques are used to facilitate this interpretation. Thus, the present work aims to propose a computer-aided detection system to collaborate with professionals in the diagnosis of breast cancer based on the analysis of scanned mammography images. Using the SURF key points algorithm and underwent a classification process with Convolutional Neural Networks (CNN) and Random Forest (RF) to generate a mass candidates dataset through the analysis of 1210 CBIS-DDSM base images. CNN presented better results reaching in training $0.06 \%$ loss and $0.97 \%$ accuracy. In the validation scenario, this proposal achieved $0.32 \%$ loss and $0.93 \%$ accuracy. For the RF model, the characteristics of the image extracted from the Hu-Moments descriptor getting an accuracy of $0.43 \% \pm 0.005$.
\end{abstract}

Key-words: breast cancer; computer vision; convolutional neural networks; 


\section{INTRODUÇÃO}

Em todo o mundo o câncer de mama é considerado um dos principais causadores de mortes em mulheres. No Brasil, a estimativa para o biênio 2018-2019 foi de 59.700 casos de câncer de mama, uma incidência de cerca de 56,33 casos a cada 100 mil mulheres [1].

A detecção precoce do câncer de mama contribui na escolha do melhor tratamento e, consequentemente, na redução da taxa de mortalidade. Métodos foram desenvolvidos e estão sendo aprimorados para a correta prevenção e detecção deste tipo de câncer, sendo a mamografia digital um dos métodos mais eficazes [2].

A mamografia digital rastreia o câncer de mama através da busca de estruturas que possam indicar a presença de anormalidades ou tumores. Estas anormalidades são de tamanho reduzido (cerca de 0,5mm de diâmetro), dificultando a visualização da lesão, exigindo grande experiência do especialista [3]. Tal dificuldade pode acarretar em diagnósticos errôneos ou tardios. Além disso, a qualidade da imagem e o tipo da lesão também afetam a análise do profissional.

Neste contexto, o avanço tecnológico mundial propõe técnicas para o correto diagnóstico do câncer de mama. Visão computacional e processamento de imagens são algumas das técnicas que tornam 0 diagnóstico menos suscetível a erros por meio da identificação precisa das anomalias [4].

Sistemas de diagnóstico ou deteç̧ão auxiliado por computador (Computer-aided diagnosis - CAD) têm o potencial de melhorar o desempenho do diagnóstico de câncer de mama [22]. Por isso, as técnicas mencionadas acima são importantes para o desenvolvimento destas ferramentas conhecidas como CAD, um sistema que auxilia os médicos na interpretação de imagens médicas, por exemplo.

É neste contexto, que a presente pesquisa tem como objetivo principal apresentar uma ferramenta CAD para auxiliar especialistas no diagnóstico do câncer de mama a partir da análise de imagens mamográficas digitais.

O artigo está dividido em cinco seções, a partir desta introdução. A segunda seção contém os trabalhos relacionados; na seção três consta o método utilizado; a quarta seção apresenta os resultados e por fim, na seção cinco, há as considerações finais.

\section{FUNDAMENTAÇÃO TEÓRICA}

Visão computacional é caracterizada como o estudo da extração de informações de uma imagem, ou seja, a construção de descrições explícitas e claras dos objetos que compõem uma imagem [5]. Já o processamento de imagens refere-se a manipulação de uma imagem digital por meio da aplicação de técnicas para análise de dados multidimensionais com utilização de um computador, onde os dados de entrada e saída deste processo são imagens digitais [6].

Sistemas CAD surgiram no final da década de 1980 e eram baseados no processamento de filmes radiológicos digitalizados. Foram fundamentalmente concebidos para trabalhar como uma segunda interpretação de exames em programas de rastreamento populacional do câncer, como por exemplo detectar nódulos e microcalcificações em mamografias [7].

Estes sistemas têm o objetivo de oferecer melhores parâmetros para a elaboração de um diagnóstico mais cuidadoso, indicando áreas suspeitas, bem como anomalias. Eles foram desenvolvidos com o intuito de minimizar os casos de falsos negativos, bem como os falsos positivos, proporcionando um importante auxílio no diagnóstico médico em diversas aplicações radiológicas, sobretudo na mamografia [8].

Há dois tipos principais de CAD, um é a deteç̧ão auxiliada por computador (Computer Aided Detection - CADe) e o outro é o diagnóstico assistido por computador (Computer Aided Diagnosis - CADx). O CADe identifica e marca as áreas suspeitas em uma imagem, eles ajudam o profissional a encontrar possíveis áreas com patologia do câncer, por exemplo. Já o CADx ajuda o profissional a decidir se um paciente deve realizar ou não uma biópsia [9].

Santos et al. [7] apontam que os sistemas CAD se expandiram e passaram a ser utilizados nas mais diferentes tarefas, principalmente após o desenvolvimento da inteligência artificial e das ferramentas de aprendizado de máquina, bem como com a utilização das técnicas de visão computacional. 


\subsection{Trabalhos Relacionados}

Várias pesquisas acadêmicas apresentam sistemas CAD para diagnósticos de variados tipos de cânceres ou outras doenças. Nesta pesquisa serão enfatizados algumas pesquisas de CAD para 0 diagnóstico de câncer.

Na pesquisa de Lee e Chen [10] os autores fazem uma análise sobre as principais técnicas utilizadas em sistemas CAD, posteriormente propõem um framework baseado na tecnologia de serviço em nuvem da Microsoft para utilização dos algoritmos desenvolvidos por diferentes pesquisadores. A proposta dos autores é que médicos possam acessar vários algoritmos, escolhendo o mais adequado para o processamento das imagens de acordo com o tipo de câncer. Por fim, os pesquisadores afirmam que o sistema não deve em nenhum momento substituir o papel do profissional de saúde responsável, servindo apenas como auxílio às atividades em busca por diagnósticos cada vez mais corretos.

Mohebian et al. [11] propuseram um sistema CAD para identificar recorrência do câncer dentro de cinco anos após o diagnóstico da doença. Para desenvolvimento do CAD os autores utilizaram uma técnica híbrida incluindo a Statistical Features Selection, Meta-heuristic Population-based e aprendizado de conjuntos (Ensemble Learning). Sobre a avaliação do CAD desenvolvido, os autores pontuam que os resultados podem ter sido tendenciosos, porque apenas uma instituição foi utilizada para amostragem. Assim, sugerem que o tamanho da amostragem deve ser maior para melhorar os dados de validação.

Miranda e Felipe [12] apresentam um CAD desenvolvido a partir da utilização da caracterização Fuzzy e da Breast Imaging-Reporting and Data System (BI-RADS). O CAD foi concebido com colaboração de especialistas e teve como resultado um sistema que sugere ao usuário uma categoria BIRADS. Ao utilizar os conceitos Fuzzy, os autores pontuam que $O$ CAD fornece funcionalidades importantes como: a entrada de dados é realizada de forma mais amigável, já que o usuário pode qualificar os descritores em graus, ou seja, está bem próximo da maneira natural como o radiologista avalia e classifica as lesões em uma imagem; e, a saída é mostrada por meio de graus de pertinência para as classes mais prováveis, dando ao usuário uma sugestão mais rica. Por fim, os pesquisadores apontam que os resultados, em relação à precisão e sensibilidade/especificidade, mostraram que a abordagem é promissora sendo um novo modo para o desenvolvimento de sistemas CAD.

$\mathrm{Na}$ pesquisa de Cheng et al. [13] os autores apresentam um sistema CADx baseado em deep learning (aprendizado profundo) para o diagnóstico diferencial de nódulos/lesões benignos e malignos, evitando os possíveis erros causados por resultados imprecisos do processamento de imagens, bem como o viés de classificação resultante de um conjunto de recursos menos robusto, envolvido na maioria dos algoritmos CADx convencionais. Os resultados mostraram um aumento significativo do desempenho do algoritmo proposto em relação aos convencionais, sugerindo que técnicas de deep learning podem potencialmente mudar o paradigma dos sistemas CADx.

Foi observado que Algoritmos de Detecção de Pontos de Interesse não são utilizados como base de conhecimento em sistemas CAD's com o objetivo principal de detecção de lesões em imagens mamográficas. Sua aplicação fica restrita apenas para extração de características das imagens que podem servir de entrada para modelos de aprendizado de máquina. Sendo assim, o método proposto e relatado no presente artigo, utiliza os algoritmos de detecção de pontos de interesse para geração de candidatos à lesão.

\section{MÉTODO PROPOSTO}

Comumente os métodos utilizados para detecção são baseados em técnicas de janela deslizante, e assim como apresentado em Agarwal et al. [14] geram uma grande quantidade de candidatos Falsos Positivos (FP) além de necessitar de um alto custo computacional e tempo de processamento. Desta forma, assim como apresentado em Oliveira [6], algoritmos de detecção de pontos de interesse apresentaram uma alternativa para a geração de candidatos à lesão, permitindo que este processo ocorra de maneira mais rápida e mais eficiente.

Neste contexto, um sistema CADe é proposto nesta pesquisa com o objetivo de auxiliar na detecção do câncer de mama. Para isso, foi utilizado como base o algoritmo de detecção de ponto de interesse chamado SURF, que foi escolhido por ter 
apresentado melhores resultados ao ser comparado com outros algoritmos da mesma classe [6]. A Figura 1 mostra como é realizado o fluxo geral do sistema proposto.

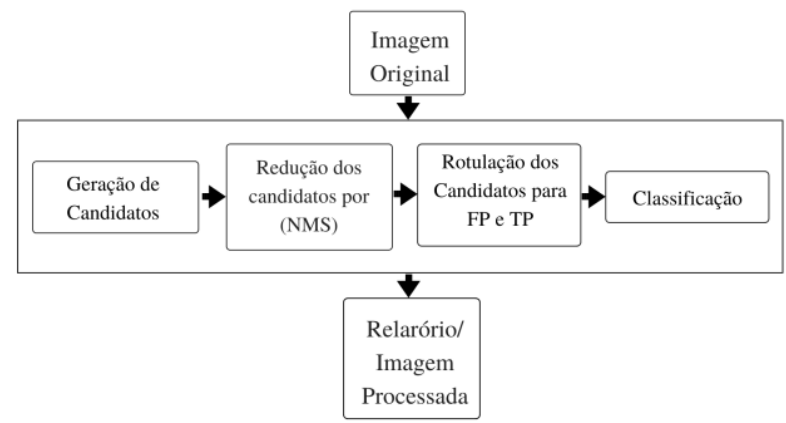

Figura 1: Fluxo da abordagem proposta.

Fonte: Os autores.

\subsection{Geração de candidatos à lesão}

Após ser realizado o carregamento da imagem no sistema, o processo de geração de candidatos à lesão é executado utilizando como base o algoritmo SURF [6]. O conjunto de candidatos contendo as ROI's passam por um processo de filtragem onde o objetivo principal é reduzir o espaço exploratório na imagem. Ao término desta etapa, é realizado um processo de análise dos candidatos restantes, esta fase visa reduzir a quantidade de candidatos considerados com alto nível de intersecção.

\subsection{Redução dos candidatos com alto nível de intersecção}

Após análise dos candidatos restantes, foi observado que muitos deles continham um alto nível de intersecção com outros demais candidatos, em alguns casos, um candidato se mantém completamente contido na região de outro.

A Figura 2 mostra um conjunto de candidatos à lesão, onde representado pela cor verde um candidato está completamente contido em outro que, para o exemplo em questão, está representado pela cor vermelha, o mesmo caso se repete em diversas vezes como é possível observar nos candidatos da cor azul.

Para reduzir o número de candidatos com alto nível de intersecção foi aplicado o algoritmo NonMaximum-Suppression (NMS) [16]. Este algoritmo tem como objetivo reduzir em apenas um candidato à objeto, um conjunto de candidatos com alta sobreposição entre si. Como entrada do algoritmo é passado uma lista de candidatos e o nível de sobreposição entre eles, o valor relacionado ao nível de sobreposição é dado através da métrica Intersecção pela União (IoU).



Figura 2: Candidatos à lesão e exemplo de sobreposição. Fonte: Os autores.

O valor do IoU é alcançado pelo resultado da divisão entre a intersecção sobre a união dos pixels entre duas janelas sobrepostas. Este valor quanto mais próximo de 1 significa que uma janela está mais alinhada sobre a outra, em termos de sobreposição [6]. Na Figura 3 é apresentado a aplicação do algoritmo NMS, onde é possível observar nas caixas em verde a redução em apenas uma janela candidata, cada grupo de candidatos com alto nível de sobreposição, assim como observado nas caixas vermelhas e azuis.

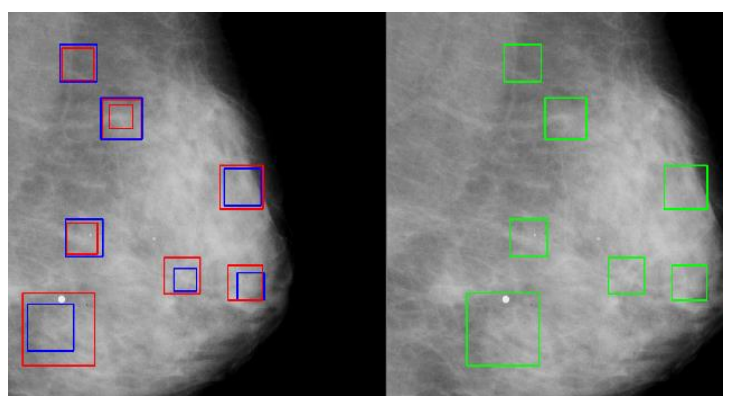

Figura 3: Aplicação do algoritmo NMS para grupos de candidatos com alto nível de sobreposição.

Fonte: Os autores.

Os candidatos resultantes após o processo de filtragem foram passados como entrada para 0 treinamento da CNN e, assim como observado no 
estado na arte, as imagens foram ajustadas para tamanho $300 \times 300$ pixels.

\subsection{Geração de candidatos Falsos Positivos FP e Verdadeiros Positivos TP}

Com base na marcação da lesão real fornecida pelos médicos foi possível criar uma base rotulada de candidatos que podem servir como entrada para algoritmos supervisionados de classificação de imagens, assim como apresentado em Agarwal et al. [14], um candidato é rotulado como positivo (candidato com lesão TP) se o pixel central da janela do candidato estiver dentro da janela da lesão real, caso contrário, é atribuído um rótulo negativo (sem massa FP).

A Figura 4 apresenta como o processo é dado, a caixa em azul representa a janela contendo a lesão real fornecida pelo médico, a caixa verde representa um candidato rotulado como verdadeiro, ou seja, com lesão, e a caixa vermelha representa um candidato considerado negativo, sem lesão.

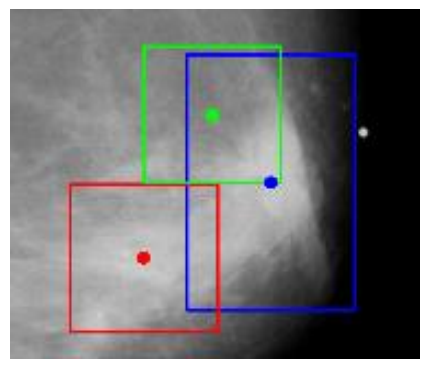

Figura 4: Geração da base de candidatos com lesão e sem lesão.

Fonte: Os autores.

\subsection{Modelos de Classificação Analisados}

Com os recentes avanços no campo da aprendizagem de máquina, o uso de Redes Neurais Convolucionais (CNNs) na análise de imagens médicas tornou-se muito encorajador [17]. Ao contrário dos métodos tradicionais de aprendizagem de máquina, onde as características das imagens são extraídas por outro algoritmo que não faz parte da composição da rede, a CNN extrai automaticamente características das imagens de entrada previamente. Durante o processo de aprendizagem, são encontrados os valores ideais para os coeficientes de convolução (pesos), por meio de uma função de custo predefinida, com base nos quais os recursos são determinados automaticamente [18]. O Treinamento e validação das CNN's são realizados por épocas e passos por época. Uma época consiste em um ciclo completo através de todos os dados de treinamento, os passos por época geralmente são definidos com base na definição de tamanho de um lote até que toda a base seja percorrida, por exemplo, se a base tiver 1.000 imagens e um tamanho de lote de 10, a época deverá conter $1.000 / 10=10$ passos.

A arquitetura da CNN utilizada para as primeiras análises foi retirado do trabalho apresentado por Sun et al. [19], a arquitetura da CNN contém 8 camadas e toda camada de número ímpar é uma camada de convolução e toda camada de número par é uma camada de agrupamento e subamostragem [19]. Para cada convolução foi utilizado 12, 8 e 6 mapas de características, e todos eles estão conectados às camadas de pool através de filtros $5 \times 5$.

Além da utilização de uma CNN para o processo de classificação dos candidatos como lesão e nãolesão, foi analisado o comportamento de uma uma técnica chamada Random Forest (RF), essa técnica é baseada em uma combinação de preditores de árvores de decisão, de modo que cada árvore depende dos valores de um vetor aleatório e com a mesma distribuição para todas as árvores da floresta [20].

\subsection{Extração de Características com Hu-Moments}

A extração de características como Hu-Moments busca recursos globais e invariantes de uma imagem. Essas características são medidas estatísticas projetadas para permanecerem constantes após algumas transformações, como rotação de objetos, escala e conversão [21]. Quando aplicado em uma imagem, o algoritmo $\mathrm{Hu}$ Moments retorna um vetor contendo 7 elementos que caracterizam a imagem, esses parâmetros são passados como entrada para RF assim como o label indicando se o candidato contém lesão ou não.

O Quadro 1 mostra como foi estruturado os dados de entrada para a RF após extração de características e rotulação dos candidatos gerados. Na primeira coluna representa a classe, sendo assim, 
0 para candidato sem lesão e 1 para candidatos com lesão, as demais colunas representam as características geradas, ao total é gerado uma matriz de 2296 linhas $\times 8$ coluna, sendo 1127 linhas para classe 0 e 1169 da classe 1.

Quadro 1: Base de treinamento gerado pelo algoritmo $\mathrm{Hu}-$ Moments.

\begin{tabular}{|c|c|c|c|c|c|c|c|}
\hline$\#$ & $F 1$ & $F 2$ & $F 3$ & $F 4$ & $F 5$ & $F 6$ & $F 7$ \\
\hline 0 & 0,0012 & 1,02 & 6,31 & 8,82 & 2,10 & 8,59 & 6,23 \\
\hline 0 & 0,0010 & 1,38 & 2,96 & 3,31 & $-1,01$ & 1,06 & $-3,11$ \\
\hline 0 & 0,0012 & 1,59 & 2,08 & 1,19 & 5,22 & $-1,82$ & 2,91 \\
\hline. & $\ldots$ & $\ldots$ & $\ldots$ & $\ldots$ & $\ldots$ & $\ldots$ & $\ldots$ \\
\hline 1 & 0,0010 & 3,86 & 9,10 & 5,02 & $-2,29$ & $-1,07$ & 3,39 \\
\hline 1 & 0,0008 & 6,28 & 1,17 & 1,13 & $-1,72$ & $-1,13$ & 3,79 \\
\hline 1 & 0,0008 & 4,56 & 1,00 & 3,16 & 1,36 & 3,60 & 1,14 \\
\hline
\end{tabular}

Fonte: Os autores.

\subsection{Base de Imagens e Ambiente Experimental}

Para realização do método proposto foram utilizadas 1210 imagens da base de imagens Curated Breast Imaging Subset of DDSM (CBISDDSM). Esta base é uma versão atualizada e padronizada da Digital Database for Screening Mammography (DDSM). Na CBIS-DDSM há 6775 casos de estudos relacionados à 6671 pacientes, totalizando 10239 imagens. Há também imagens digitais de mamografia capturadas de ângulos diferentes.

Já a base DDSM contém 2.620 casos de estudos de pacientes com casos normais, benignos $e$ malignos com informações patológicas diagnosticadas por médicos. Entretanto, algumas informações são limitadas, especificamente as anotações de ROI (Region of Interest - ROI), enquanto outras informações são de difícil acesso [15]. A base CBIS-DDSM inclui um subconjunto dos dados DDSM selecionados por profissionais de saúde treinados. As imagens foram descompactadas e convertidas para o formato PNG. A base possui Imagens Ouro e imagens das regiões de interesse atualizadas.
Em relação ao ambiente experimental, o algoritmo implementado de geração e filtragem de candidatos foi desenvolvido na linguagem de programação Python, com auxílio da biblioteca OpenCV em um computador com processador i7 e 8GB de memória RAM. O sistema foi desenvolvido através do framework Django, que utiliza Python como back-end, também foi utilizado a biblioteca Bootstrap para criação das telas, bem como o uso das linguagens de marcação HTML5 e CSS, além de JavaScript para execução de atividades de interação com o usuário.

\section{RESULTADOS}

\subsection{Redução dos Falsos Positivos}

O processo de execução do algoritmo NMS tem como objetivo remover da base os candidatos que possuem alto nível de intersecção sobre outros candidatos. Após a execução do processo de filtragem dos FP a partir da aplicação da técnica do NMS, em comparação aos números de candidatos gerados pelo método apresentado por Oliveira [6], houve uma redução de $28.55 \%$ no número de candidatos por cada imagem. Ao término do processo, restaram uma média de 46,40 candidatos por imagem com desvio padrão de 29,43.

Esses candidatos restantes serviram de entrada para os processos de classificação, na primeira análise foi utilizado uma CNN, na segunda análise foi utilizado uma RF, os resultados da aplicação dessas técnicas estão descritas abaixo.

\subsection{Resultados dos experimentos de classificação utilizando CNN}

Foram realizados 4 experimentos utilizando a CNN, como o aprendizado é supervisionado, foi necessário gerar a base de treino e teste para cada classe de imagens, ou seja, imagens com lesão e as imagens sem lesão, cada um deles está descrito abaixo:

- Primeiro experimento: Neste experimento foram utilizados como base de treinamento, teste e validação, apenas as ROI's das marcações fornecidas previamente pelos 
médicos para imagens com lesão e, para geração da base das imagens sem lesão, foram geradas a partir de candidatos extraídos de forma aleatória das imagens, neste experimento a ROI contendo a lesão foi retirada da possibilidade de seleção de um candidato sem lesão. Aqui foram utilizadas 1127 imagens para base sem lesão e 1169 ROI's com lesão;

- Segundo experimento: Neste experimento foram geradas imagens para base de treinamento, teste e validação utilizando a técnica apresentada na seção 3.3. Devido a essa aplicação o número de imagens consideradas com lesão subiu para 4551, o mesmo número foi gerado para candidatos extraídos das imagens e considerados sem lesão;

- Terceiro experimento: Neste experimento a base utilizada para treinamento, teste e validação foi a mesma utilizada no segundo experimento, aqui as configurações de épocas e passos por épocas da CNN foram definidas como as mesmas utilizadas no primeiro experimento;

- Quarto experimento: Neste experimento a base utilizada foi a mesma gerada para o segundo experimento alterando apenas a quantidade de épocas e passos por épocas da CNN.

Em todos os experimentos a base de imagens foi dividida em $75 \%$ para treinamento e $25 \%$ para teste. Todos os resultados são apresentados no Quadro 2.

Quadro 2: Resultados dos experimentos utilizando CNN.

\begin{tabular}{|c|c|c|c|c|c|c|c|}
\hline \multirow[t]{2}{*}{ \# } & \multirow[t]{2}{*}{$\begin{array}{c}\# \\
\text { Imagens }\end{array}$} & \multirow[t]{2}{*}{$\begin{array}{c}\# \\
\text { Épocas }\end{array}$} & \multirow[t]{2}{*}{$\begin{array}{c}\# \\
\text { Passos }\end{array}$} & \multicolumn{2}{|c|}{ Treino } & \multicolumn{2}{|c|}{ Validação } \\
\hline & & & & loss & acc & $\begin{array}{c}\text { val_I } \\
\text { oss }\end{array}$ & $\begin{array}{l}\text { val_ } \\
\text { acc }\end{array}$ \\
\hline 1 & 2296 & 10 & 800 & 0,06 & 0,97 & 0,32 & 0,93 \\
\hline 2 & 9102 & 30 & 3000 & 0,01 & 0,99 & 0,58 & 0,93 \\
\hline 3 & 9102 & 10 & 800 & 0,67 & 0,59 & 0,71 & 0,53 \\
\hline 4 & 9102 & 50 & 2000 & 0,14 & 0,94 & 1,95 & 0,62 \\
\hline
\end{tabular}

Fonte: Os autores.
No Quadro 2 a primeira coluna representa qual o número do experimento realizado, na segunda coluna apresenta a quantidade de imagens utilizadas, na terceira coluna apresenta o número de épocas aplicadas, na quarta coluna o número de passos aplicados por época, as colunas cinco e seis, apresentam os resultados dos experimentos no treinamento e na validação em valores de perda e acurácia. A Figura 5 apresenta 0 gráfico de convergência do quarto experimento em relação ao treinamento do modelo. Foi possível observar que a partir da época 10 o modelo começa a aprender mais e errar menos na proporção em que cresce o número de épocas.

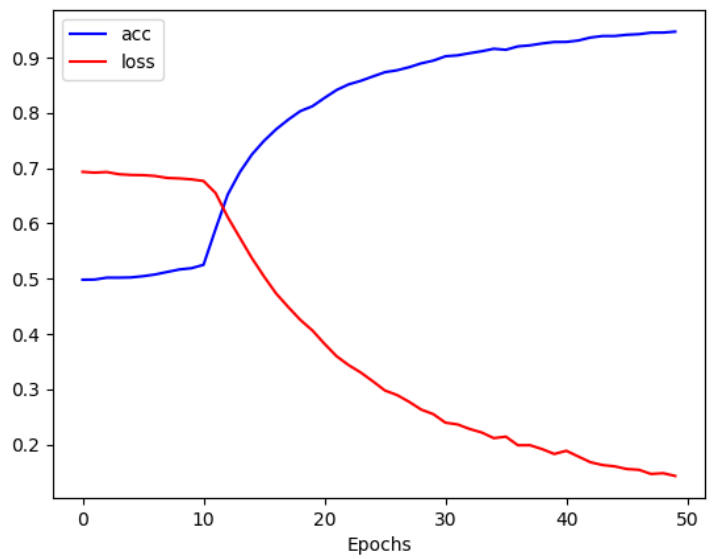

Figura 5: Curva de convergência do treinamento para o quarto experimento com 50 épocas e 2000 passos por época.

Fonte: Os autores.

O mesmo não ocorre no processo de validação, na Figura 6 é possível observar que o modelo na época 10 começa um leve processo de queda na perda e aumento na acurácia, porém em poucas épocas adiante o modelo perde mais e aprende menos, a medida que cresce o número de épocas. Em comparação com o gráfico de treinamento é possível inferir que, a partir da época 10, houve um processo de sobreajuste (overfitting), ou seja, o modelo perdeu a capacidade de generalização ficando super ajustado para apenas uma classe.

Fica ainda mais evidente o processo de overfitting ao observar na Figura 7 quando é apresentado no mesmo gráfico os valores de acurácia no treinamento e na validação. É novamente notório que a partir da décima época o modelo deixa de aprender quando the é fornecido novos dados na validação, ou seja, perde a capacidade de 
generalização tornando especialista apenas para um conjunto de dados conhecidos.

O mesmo processo ocorre tanto nos experimentos 2 e 3 assim como observado no Quadro [2].

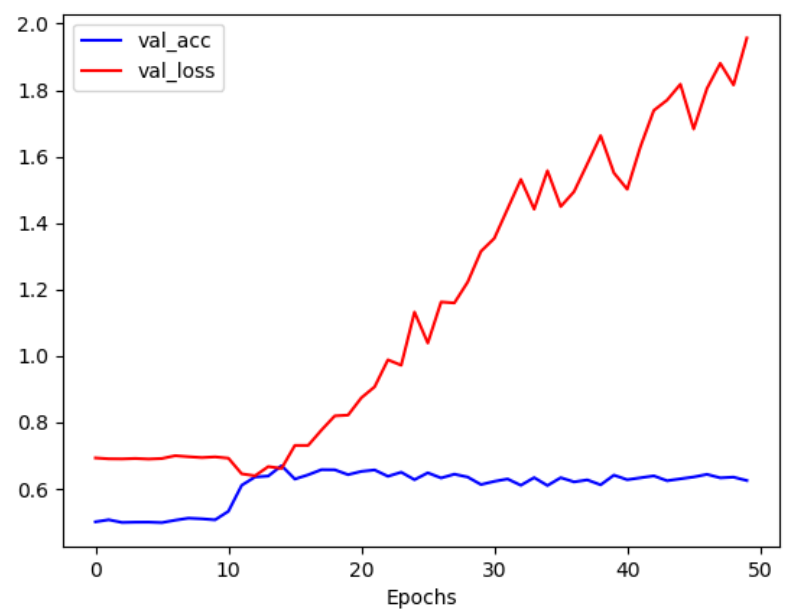

Figura 6: Curva de convergência na validação para o quarto experimento com 50 épocas e 2000 passos por época.

Fonte: Os autores.

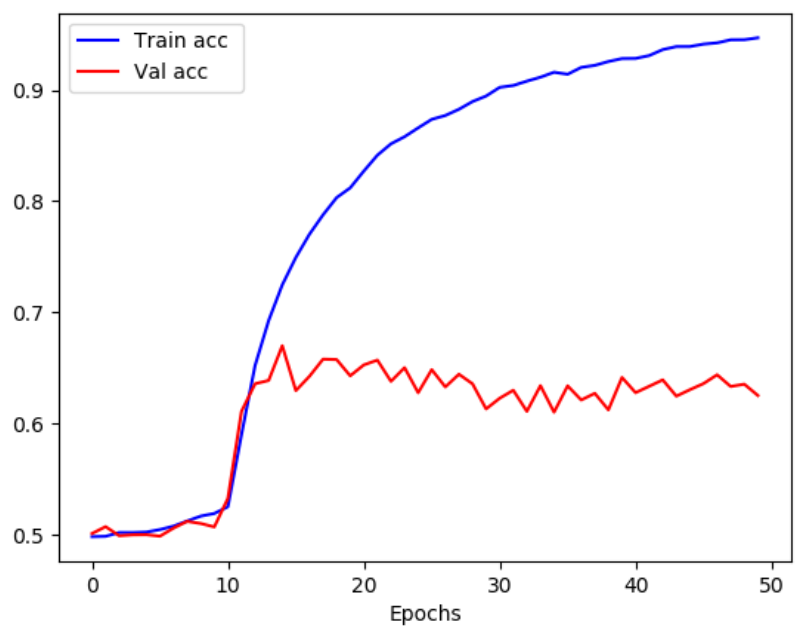

Figura 7: Resultado da acurácia no treinamento e na validação dado o aumento de épocas.

Fonte: Os autores.

\subsection{Resultados dos experimentos de classificação utilizando Hu- Moments e RF}

A base de imagens utilizada para extração de características por meio do descritor Hu-Moments foi a mesma aplicada no primeiro experimento com CNN explicado na Seção 4.2. A base foi dividida em $80 \%$ para treinamento e $20 \%$ para teste, a profundidade da RF foi definida por 10 . Foi aplicado o processo de validação cruzada para 4, 7 e 10 k-folds.

Para comprovação estatística, o processo foi executado 30 vezes para cada grupo de $k$-folds. A técnica shuffle foi aplicada para certificar que o processo de reprodução aleatória de dados tenha o objetivo de reduzir as variações e garantir que o modelo permaneça geral e se ajuste menos. Os resultados estão descritos no Quadro 3.

Quadro 3: Resultados dos experimentos utilizando RF..

\begin{tabular}{|c|c|c|}
\hline \# K-Fols & $\begin{array}{c}\text { Acurácia Média } \\
(\%)\end{array}$ & Desvio Padrão \\
\hline 4 & 0,42 & $\pm 0,012$ \\
\hline 7 & 0,42 & $\pm 0,007$ \\
\hline $\mathbf{1 0}$ & $\mathbf{0 , 4 3}$ & $\mathbf{0 , 0 0 5}$ \\
\hline
\end{tabular}

Fonte: Os autores.

$\mathrm{Na}$ primeira coluna apresenta o valor do $\mathrm{k}$ atribuído na validação, na segunda coluna a média na acurácia após as 30 execuções e por fim o desvio padrão para cada experimento. É possível observar que para a configuração de $10 \mathrm{k}$-folds o modelo teve uma melhor acurácia além de uma taxa de medida de dispersão próximo a zero mostrando que conjunto de dados é uniforme.

\subsection{A ferramenta LUISA}

A ferramenta desenvolvida tem como principal objetivo processar imagens de mamografias e fornecer para os profissionais de saúde propostas de candidatos a lesão com alta taxa de exatidão, para que o processo de diagnóstico se torne ainda mais rápido e preciso. Em nenhum momento a ferramenta pretende anular a ação do profissional, ela pretende servir apenas como auxílio às atividades rotineiras de detecção e posteriormente diagnóstico do câncer de mama, além de oferecer um suporte ao gerenciamento das informações entre profissionais de saúde e pacientes durante todo o processo.

Ainda, o sistema fornece a capacidade de gerenciamento de cadastro de médicos e pacientes além de agendamento de consultas e análise das 
imagens no mesmo ambiente. A Figura 8 mostra como é apresentado a tela principal do sistema.

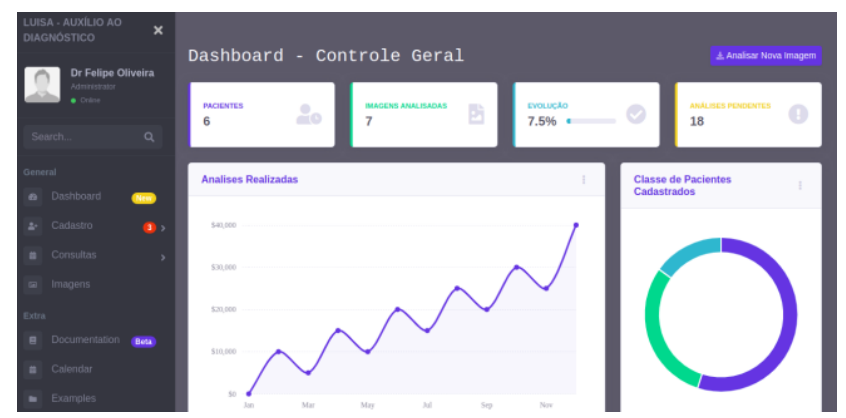

Figura 8: Tela inicial do sistema contendo as informações principais dos pacientes.

Fonte: Os autores.

A partir da tela principal o médico pode observar dados sobre seus pacientes e, por meio de gráficos, ver informações importantes sobre as rotinas de análises. A Figura 9 mostra como é apresentada a tela para carregamento da imagem no sistema. Neste momento, o médico seleciona um paciente e atribui a ele a sua respectiva imagem, o sistema faz o processamento e redireciona para uma tela com a lista de imagens carregadas.

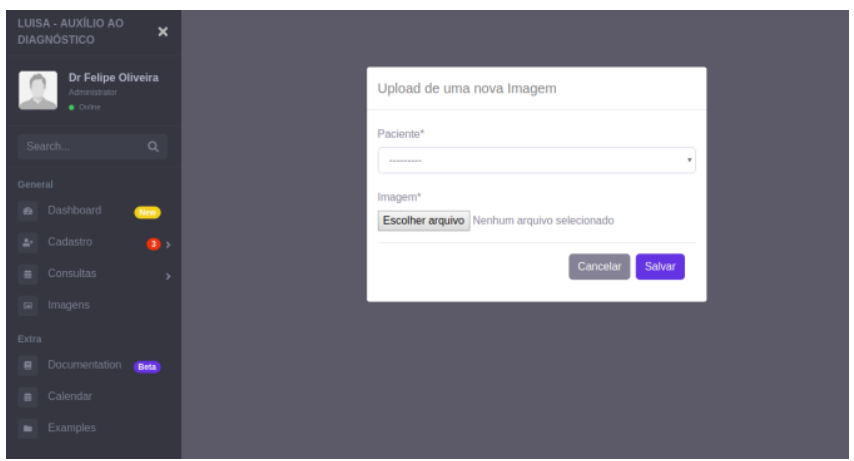

Figura 9: Tela de carregamento das imagens dos pacientes.

Fonte: Os autores.

A Figura 10 mostra como é apresentada a tela com a lista de imagens em análises, ao clicar em mais detalhes o médico envia para o sistema a solicitação para apresentação de candidatos à lesão que o modelo processou.

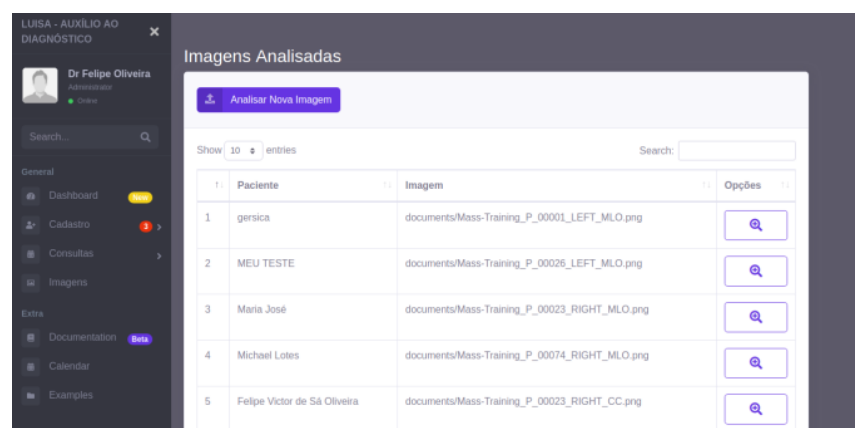

Figura 10: Tela com a lista de imagens carregadas no sitema.

Fonte: Os autores.

A Figura 11 mostra o resultado da análise e processamento da imagem realizada pelo sistema, são apresentadas 3 versões da mesma imagem, na primeira mostra a imagem original, a segunda em vermelho mostra a imagem contendo todos os candidatos gerados e por último em azul os candidatos restantes a partir do processo de filtragem por sobreposição.
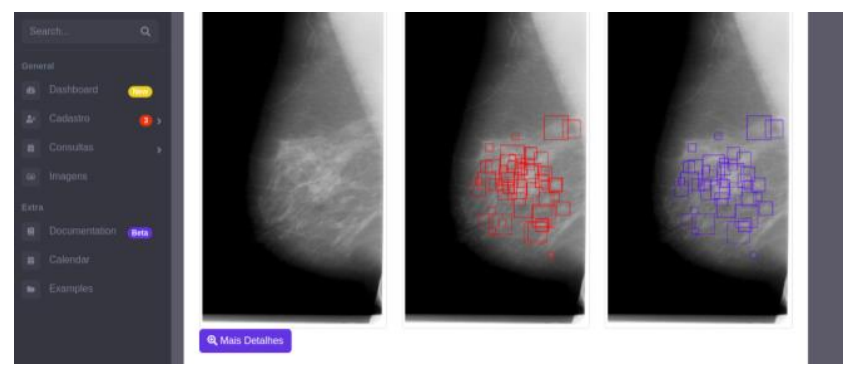

Figura 11: Tela de apresentação dos candidatos gerados pelo sistema.

Fonte: Os autores.

Ainda, o médico pode optar por ter mais detalhes visuais da imagem original, a ferramenta possibilita que o usuário clicando em mais detalhes abra uma tela contendo uma lupa, facilitando o processo de visualização e posteriormente aplicação de uma marcação na imagem feita manualmente, fornecendo um novo candidato a ser analisado ou então realizando a detecção da lesão. A Figura 12 mostra a tela de mais detalhes da imagem. 




Figura 12: Tela mostrando a ferramenta de análise mais detalhada das imagens.

Fonte: Os autores.

\section{CONSIDERAÇÕES FINAIS}

O trabalho desenvolvido propôs uma ferramenta CAD para auxiliar profissionais de saúde no diagnóstico de câncer de mama por meio de imagens de mamografias digitalizadas. O sistema utilizou um novo método para seleção de candidatos à região suspeita de lesão baseado na utilização de algoritmos de detecção de pontos de interesse. Para filtragem dos candidatos considerados Falsos Positivos foi aplicado o algoritmo NMS que alcançou uma taxa média de $28.55 \%$ de redução por imagem.

Ainda, para comprovar a eficiência do método, os candidatos restantes passaram por um processo de classificação utilizando os modelos CNN e RF. O primeiro modelo apresentou melhores resultados para a base de dados contendo 2296 imagens, alcançando no treinamento $0.06 \%$ de perda e $0.97 \%$ acurácia.

No processo de validação, o modelo também se comportou melhor que todas as outras configurações testadas com $0.32 \%$ de perda e $0.93 \%$ de acurácia. O modelo RF também foi testado, neste, as características das imagens foram extraídas a partir do descritor Hu-Moments e serviram de entrada obtendo uma acurácia de $0.43 \% \pm 0.005$.

O sistema apresentado mostrou que tem potencial para servir como auxílio aos profissionais de saúde, contribuindo na redução de possíveis erros durante o processo de diagnóstico do câncer de mama. Além de servir como suporte no gerenciamento de informações entre médicos e pacientes geradas durante todo o processo.

Como trabalhos futuros pretende-se aplicar outras técnicas de aprendizado de máquina em busca de melhores resultados no processo de 82 classificação. Ainda, realizar mais experimentos alterando configurações dos modelos aplicados na presente pesquisa com o objetivo de contornar o problema do overfitting na CNN. Por fim, realizar uma validação da aplicação junto a profissionais de saúde para verificação da eficiência e usabilidade do sistema proposto.

\section{AGRADECIMENTOS}

Agradecemos a FITec, SECTI, CMA-Parqtel, Universidade de Pernambuco e FACEPE pelo apoio intelectual fornecido e o subsídio necessário para o desenvolvimento da pesquisa.

\section{Referências}

[1] INSTITUTO NACIONAL DO CÂNCER. Incidência do Câncer no Brasil. Disponível em:<http://www1.inca.gov.br/estimativa/2018/ >. Acesso em 06 nov. 2019.

[2] TORTORA, G. J.; DERRICKSON, B. Corpo Humano: Fundamentos de Anatomia e Fisiologia. Artmed Editora, 2016.

[3] PIRES, S. R.; MEDEIROS, R. B.; SCHIABEL, H. Banco de imagens mamográficas para treinamento na interpretação de imagens digitais. Radiologia Brasileira, 2004.

[4] CORDEIRO, F. R.; SANTOS, W. P. d.; SILVAFILHO, A. G. Analysis of supervised andsemisupervised growcut applied to segmentation of masses in mammography images. Computer Methods in Biomechanics and Biomedical Engineering: Imaging \& Visualization, Taylor \& Francis, v. 5, n. 4, p. 297-315, 2017.

[5] BROWN, C. M. Computer vision and natural constraints. Science, v. 224, n. 4655, p. 1299-1305, 1984.

[6] OLIVEIRA, F. V. S. Uma abordagem para seleção de candidatos à região de lesão em imagens de mamografia digitalizadas utilizando algoritmos de detecção de pontos de interesse. 2019. 92 f. Dissertação (Mestrado) - Curso de Mestrado em Informática Aplicada, Universidade Federal Rural de Pernambuco, Recife, 2019. 
[7] SANTOS, M. K. et al.; Inteligência artificial, aprendizado de máquina, diagnóstico auxiliado por computador e radiômica: avanços da imagem rumo à medicina de precisão. Radiologia Brasileira, 2019.

[8] PINHO, S. R. A. Apoio ao Diagnóstico de Imagens de Mamografia por Técnicas Computacionais. 2015. 76 f. Dissertação (Mestrado) - Curso de Mestrado em Engenharia Biomédica, Faculdade de Engenharia da Universidade do Porto, Universidade do Porto, Porto, 2015.

[9] PORTO, F. N. Análise de sensibilidade de um sistema CAD para mamografia digital. 2010. 69 f. Dissertação (Mestrado) - Curso de Mestrado em Engenharia Elétrica, Escola de Engenharia, Universidade Federal de Minas Gerais, Belo Horizonte, 2010.

[10] LEE, H.; CHEN, Y. P. Image based computer aided diagnosis system for cancer detection. Expert Systems with Applications, v. 42, n. 12, p. 5356-5365, 2015.

[11] MOHEBIAN, M. R. et al. A hybrid computer-aided-diagnosis system for prediction of breast cancer recurrence (HPBCR) using optimized ensemble learning. Computational and structural biotechnology journal, v. 15, p. 75-85, 2017.

[12] MIRANDA, G. H. B.; FELIPE, J. C. Computer-aided diagnosis system based on fuzzy logic for breast cancer categorization. Computers in biology and medicine, v. 64, p. 334-346, 2015.

[13] CHENG, Jie-Zhi et al. Computer-aided diagnosis with deep learning architecture: applications to breast lesions in US images and pulmonary nodules in CT scans. Scientific reports, v. 6, p. 24454, 2016.

[14] AGARWAL, Richa et al. Automatic mass detection in mammograms using deep convolutional neural networks. Journal of Medical Imaging, v. 6, n. 3, p. 031409, 2019.

[15] LEE, R. S. ET al.; A curated mammography data set for use in computer-aided detection and diagnosis research. Scientific data, Nature Publishing Group, v. 4, p. 170177, 2017.

[16] ROTHE, R.; GUILLAUMIN, M.; VAN GOOL, L. Non-maximum suppression for object detection by passing messages between windows. Asian conference on computer vision. Springer, Cham, 2014. p. 290-306.

[17] AGARWAL, R. et al. Mass detection in mammograms using pre-trained deep learning models. 14th International Workshop on Breast Imaging (IWBI 2018). International Society for Optics and Photonics, 2018. p. $107181 \mathrm{~F}$.

[18] YEMINI, M.; ZIGEL, Y.; LEDERMAN, D. Detecting Masses in Mammograms using Convolutional Neural Networks and Transfer Learning. 2018 IEEE International Conference on the Science of Electrical Engineering in Israel (ICSEE). IEEE, 2018. p. 1-4.

[19] SUN, W.; ZHENG, B.; QIAN, W. Computer aided lung cancer diagnosis with deep learning algorithms. Medical imaging 2016: computer-aided diagnosis. International Society for Optics and Photonics, 2016. p. $97850 Z$.

[20] BREIMAN, L. Random forests. Machine learning, v. 45, n. 1, p. 5-32, 2001.

[21] OTINIANO-RODRIGUEZ, K. C. ET al. Zernike moments for sign language recognition. Proceedings of international conference on image processing, computer vision, and pattern recognition. 2012. p. 1-5.

[22] YEMINI, M.; ZIGEL, Y.; LEDERMAN, D. Detecting Masses in Mammograms using Convolutional Neural Networks and Transfer Learning. 2018 IEEE International Conference on the Science of Electrical Engineering in Israel (ICSEE). IEEE, 2018. p. 1-4. 